\title{
SANS STUDY OF HYBRID SILICA AEROGELS \\ UNDER "IN SITU” UNIAXIAL COMPRESSION
}

\author{
N. de la Rosa-Fox ${ }^{1}$, V. Morales-Flórez, J.A. Toledo-Fernández, \\ M. Piñero ${ }^{a}$, L. Esquivias ${ }^{b}$ and U. Keiderling ${ }^{c}$ \\ Dpto. Física Materia Condensada. Facultad Ciencias, \\ ${ }^{a}$ Dpto. Física Aplicada. CASEM \\ Universidad de Cádiz \\ Avda./ República Saharaui, s/n. 11510 Puerto Real (Cádiz) \\ ${ }^{\mathrm{b}}$ Dpto. Física Materia Condensada. \\ Universidad de Sevilla \\ SPAIN \\ ${ }^{\mathrm{c} B e r l i n}$ Neutron Scattering Center. HMI. Berlin (Germany)
}

Abstract.

We have modified the inorganic silica network of aerogels with polydimethylsiloxane (PDMS), a hydroxyl-terminated polymer, to obtain an ORganic MOdified SILicate (ORMOSIL). Reactions were assisted by high-power ultrasounds. The resulting gels were dried under supercritical conditions of the solvent to obtain a monolithic sono-aerogel. The mechanical behaviour of these aerogels can be tuned from brittle to rubbery as a function of the organic polymer content. In order to determine the links between the mechanical behaviour and modifications made to the microstructure, SANS (small-angle neutron scattering) experiments were carried out. To measure the intensities under "in situ" uniaxial compression of the aerogel, a specific sample-holder was built. Under uniaxial compression the 2D-diagrams were significantly anisotropic (butterfly pattern), indicating the rearrangement of the polymer. The form factor of these aerogels is described well by twocorrelation length, small microporous silica clusters surrounded by entangled polymer chains of $6 \mathrm{~nm}$ average size (blobs), which form a larger secondary level of agglomerates governed by the "frozen-in" elastic constraints.

PACS: 51.12.Ex, 61.41.-e, 62.20.-x, 82.33.Ln

Keywords: Aerogels A120; Mechanical Properties M120; Small Angle Neutron Scattering S250; Sol-Gel Method S290

\footnotetext{
${ }^{1}$ Contact author: nicolas.rosafox@uca.es
} 


\section{1.- Introduction}

TEOS (tetraethoxysiloxane), a silicon alkoxide, polycondenses easily to form an entangled 3D network. The complex structure of hybrid organic/inorganic aerogels can be described basically as the TEOS inorganic phase covalently bonded to an organic polymer chain to form a copolymer. Some authors have reported the existence of a two-correlation length function in PDMS based gels, in which the larger value is a consequence of the elastic constraints produced by the polymer cross-links $[1,2]$. Recently, Schaefer et al. found similar results in silica-reinforced elastomers [3] as well as in highly cross-linked polysilsesquioxane-based hybrids [4]. They interpret the structure as a three level hierarchical arrangement, consisting of primary particles clustered into aggregates that further cluster into agglomerates.

Several studies focus on the sol-gel method processed materials, both hybrid xerogels [5] and aerogels [6]. In the present work, the aerogels are prepared with the assistance of high power ultrasounds in the liquid state before gelation to produce "sono-aerogels" [7]. This process is known as cavitation and proceeds as follows. When a liquid is exposed to ultrasound, the waves create bubbles. Sound waves deform the bubbles, causing them to grow, then to contract and eventually to implode under adiabatic conditions. With implosion, extreme temperature and pressure are produced that speed reactions. Every collapsing bubble is a microreactor in itself, accelerating reactions unheard-of in traditional chemistry [8], which in

some cases also provoke the emission of light or sonoluminescence [9]. The acoustic multibubble cavitation via hot spot formation seems to be responsible for the homogeneous, small size of the silica clusters and microporosity in these hybrid sono-aerogels [10].

From a macroscopic mechanical point of view, the behaviour of these hybrid aerogels ranges from brittle solids to rubbery elastomers according to the polymer content [11]. As the polymer interlinks the silica clusters, the aerogel hardness and elastic modulus drop sharply. 
These hybrid aerogels are viscoelastic in regard to the time dependence of the stress at fixed strain (relaxation) and of the strain at fixed stress (creep) [12].

Small-angle neutron scattering enables the microstructure of nanostructured materials to be characterised on the 1 to $100 \mathrm{~nm}$ length scale [13]. The main objective of this work is to elucidate the internal structure responsible for the drastic changes in the mechanical behaviour of these materials.

\section{Experimental}

\subsection{Sample preparation}

Hybrid silica aerogels were synthesized by the classical sol-gel method by means a two-step procedure. First TEOS (tetraethoxysiloxane), as the inorganic phase, was partially hydrolyzed understoichiometrically with acid water $(\mathrm{pH} \sim 1)$ in a molar ratio of TEOS: $\mathrm{H}_{2} \mathrm{O}$ of 1:0.84. In this step the solution received an ultrasonic energy dose of $320 \mathrm{~J} \mathrm{~cm}^{-3}$, and the resulting mixture was a transparent and homogeneous solution. In the second step, PDMS (polydimethylsiloxane), as the organic phase, was added to complete the hydrolysis reaction with a molar ratio TEOS: $\mathrm{H}_{2} \mathrm{O}$ of 1:3.16, and once again $320 \mathrm{~J} \mathrm{~cm}^{-3}$ of ultrasound energy was applied. Several samples with different PDMS content were prepared. The liquid sol was kept in hermetically sealed container until gelification. The gel was immersed in ethanol for the aging process and to expel the residual water from the pores.

After one week of aging, the gel was placed in an autoclave and the pore liquor was ventedoff above the supercritical conditions of ethanol $\left(240^{\circ} \mathrm{C}, 63 \mathrm{bar}\right)$. The supercritical state was attained by slow heating $\left(1^{\circ} \mathrm{C} / \mathrm{min}\right)$ in order to minimize the ethanol thermal expansion coefficient, which is much greater than that of the wet silica gel network. Then the heating produces the evaporation of the additional volume of ethanol, which then permits the supercritical pressure and temperature $\left(255^{\circ} \mathrm{C}, 85 \mathrm{bar}\right)$ the vapour-liquid equilibrium curve is never crossed, to avoid capillary pressures on the adjacent pore. Provided this is done, the 
structure does not collapse and the aerogel retains its original shape. As a provision for the mechanical test, the samples were made as cylinders of approximately $18 \mathrm{~mm}$ length and 8 mm diameter. Bulk density was calculated by weighing the sample with a well defined geometry. The samples were labelled (PDMSX) with $\mathrm{X}$ being the percent by weight of organic polymer in relation to the total silica content.

\subsection{SANS measurements.}

SANS measurements were carried out at room temperature on the V4 workstation at the Berlin Neutron Scattering Center of the HMI (Berlin). A neutron wavelength of $0.602 \mathrm{~nm}$, at three sample-detector distances 1, 4 and $16 \mathrm{~m}$, was used to cover a $q$-range from 0.036 to 3.6 $\mathrm{nm}^{-1}$. Data were corrected using the software package available at HMI [14]. In these complex systems the contrast factor is a crucial parameter. By this procedure, scattering length density was estimated by weighting the corresponding inorganic silica to the polymer content monomer, considering the contrast in relation to the voids. Anisotropic data were averaged by selected angular sectors in the perpendicular and parallel directions with respect to the applied load.

We have developed a device (a physical tool) for applying "in situ" mechanical deformation by uniaxial compression to the samples for SANS or SAXS experiments, to investigate the structural changes in the hybrid aerogels submitted to a mechanical stress in real time. The sample is placed between two parallel horizontal plates, the upper one being controlled by a micrometric screw (1mm per turn), according to the scheme depicted in Fig 1. The lower plate is initially free to find the best position for the sample in the centre of the beam. Then, the lower plate is locked in position. The sample is deformed by turning the screw clockwise, which pushes the upper plate downwards thus compressing the sample. By the deformation in mm we know the applied load. The lateral guides in the apparatus were carefully aligned in 
order to assure the parallelism between the two plates. The sample is placed between the plates in the centre, immediately beneath the loading screw.

\subsection{SANS data reduction}

Given the difficulty of obtaining the scattering length density (SLD) in these complex systems, we have corrected it by using the geometrical invariant $\left(\boldsymbol{I} \cdot \boldsymbol{q}^{2} \boldsymbol{v} \boldsymbol{s} \cdot \boldsymbol{q}\right)$ and the experimental bulk densities. The Porod law $\left(\mathbf{I} \cdot \mathbf{q}^{\mathbf{4}} \mathbf{v s . q ^ { 4 }}\right)$ was used to calculate the surfacevolume ratio and the scatterer particle size, bearing in mind the chord analysis [15]. Guinier plots of $\ln \mathbf{I}$ vs. $\mathbf{q}^{2}$ provide the gyration radius, as $\boldsymbol{R}_{G}=\sqrt{\mathbf{3} \cdot|\mathbf{s l o p e}|}$, which is considered as the mean correlation length of the distribution of the solid phase or of the pore. Debye plots of $(\sqrt{\mathbf{I}})^{-\frac{1}{2}}$ vs. $\mathbf{q}^{2}$ allow the elementary particle size a to be estimated if a linear part exists in which $a=\sqrt{\text { slope/intercept }}[16]$.

\section{Results}

\subsection{Unloaded samples.}

Fig. 2 shows the SANS experimental intensities of hybrid aerogels with different polymer content. Several features can be discerned; first, an increase of the intensity is apparent towards the low q-side. Then wide plateaus appear with Guinier radii ranging from 5 to $8 \mathrm{~nm}$ as the polymer content increases from 10 to $50 \mathrm{wt} . \%$, as displayed in the inset of Fig. 2. The plateaus are followed by power law decay, with a slope increasing with the polymer content, up to a maximum value of -2.5 . The final parts of the curves are dominated by the incoherent scattering due to the ${ }^{1} \mathrm{H}$ atoms of the organic polymer in the methyl radicals. Another parameter calculated was the Debye correlation length in the linear part of the standard Debye plot that gives the elementary particle size; in all cases values of around $1 \mathrm{~nm}$ average size were obtained. 
Considering all the TEOS and the PDMS totally copolymerized, the values of the matrix scattering length density (SLD) were smaller than the theoretical ones. We have taken a weighted average of each phase content in order to calculate the coherent scattering length of the hybrid aerogel, using for $\mathrm{SiO}_{2}, \mathrm{~b}_{\mathrm{coh}}=15.7610^{-13} \mathrm{~cm}$ and for the polymer monomer ($\left.\mathrm{SiO}\left(\mathrm{CH}_{3}\right)_{2}-\right)_{\mathrm{n}}, \mathrm{b}_{\mathrm{coh}}=0.80210^{-13} \mathrm{~cm}$. In all of the cases a decrease in the SLD of about $15 \%$ is produced for the unloaded samples, but the loaded samples do not show any particular trend. When the surface volume ratio values, from Porod limit saturation, are compared to those obtained by gas physisorption (BET) in a previous work [17], the values obtained by the SANS data show higher values but the same feature.

\subsection{Loaded samples.}

When the aerogel is submitted to uniaxial compression a butterfly pattern appears in the perpendicular direction to the load, as illustrated in Fig. 3 by the 2D-diagram directly taken from the detector. A decrease of the intensity in the parallel direction to the load (from top to the bottom) can be discerned. The normalized scattering curves have two different trends in each direction, perpendicular and parallel to the load, as shown in Fig. 4a) for the sample PDMS40 in the perpendicular direction and Fig. 4b) for the PDMS30 aerogel in the parallel direction. Thus, in the perpendicular direction, the loaded and unloaded samples show the same characteristics. However, an increase of the incoherent contribution is observed, together with an increase of the intensity towards the low q-side.

In contrast, in the parallel direction, there is an appreciable decrease of the intensity towards the low q-side. In fact, a slight increase of the Guinier radius is produced in the perpendicular direction, but a significant decrease is apparent in the parallel direction. The calculated values are shown in Table I for the PDMS40 sample.

Further, the calculated Debye correlation length shows a slight reduction from 2.3 to $1.9 \mathrm{~nm}$ as the applied load increases (inset of Fig. 4b). The scattering length density and surface 
volume ratio in the loaded samples present similar values and variation as in the case of the unloaded samples.

\section{Discussion}

In Fig. 2 a first shoulder at low-q $\left(0.04 \mathrm{~nm}^{-1}\right)$ is produced by the long range characteristic length governed by the "frozen-in" elastic constraints, which act between topologicallyconnected polymer cross-links. A shorter range correlation length at $\mathrm{q}=0.3 \mathrm{~nm}^{-1}$ in the plateau corresponds to the size of the blobs, which can be considered to be composed of a nucleus of the silica cluster surrounded by the polymer distributed as a random coil (RC); the average size of the blob can be identified with the Guinier radius. This supposition is supported by the agreement between the results of non-linear fit of the experimental curves and a mixed Gaussian random coil form factor $[18,19]$. On the other hand, the wide Guinier region indicates the homogeneous distribution of these blobs as scatterers. As the polymer content increases, the blobs grow as a consequence of the entanglement of the polymer chains.

The aggregates of the entangled polymer chains surrounding the silica clusters (the blobs) form mass fractal units, their dimension $\mathrm{D}=2.5$ being characteristic of a cross-linked random coil structure. Although the aggregate does not strictly fulfil the conditions of self-similarity on different length scales to be considered a fractal object, it can be viewed as an object that does not fill the space totally. Moreover, this result suggests a kinetic growth mechanism based on the monomer-cluster diffusion limited aggregation (DLA) [20] that has a fractal dimension of 2.5 .

Further information about the interface and its surface shape is masked by the incoherent scattering. However, the Debye plot allows the correlation length to be taken as the elementary particle size, as a consequence of the higher contrast of the silica in relation to the organic polymer. This supposition is based on the fact that the Debye correlation function is exponential $\gamma(\mathbf{r})=\exp (-\mathbf{r} / \mathbf{a}), \mathbf{a}$ being the correlation length [21]. The smaller values of the 
SLD corrected by the geometrical invariant indicate that the copolymerization reaction between organic and inorganic phases has been incomplete [22]. However, the systematic variation with the polymer content indicates the equilibrium of both phases to form a good hybrid material.

The higher values of the surface volume ratio in relation to those obtained by gas physisorption can be interpreted in two ways: first in the aggregation of such blobs some closed porosity inaccessible to the nitrogen molecules may exist, but such pores can be "seen" by the neutrons. Another interpretation is that the intricate entanglement of the polymer crosslinks hinders the access of the nitrogen; this second reason is supported by a slight decrease of the surface volume ratio when the load is increased.

The butterfly pattern informs about the deformation caused by the uniaxial compression; whereas in the perpendicular direction to the load, the behaviour is similar to the non-loaded sample (Fig. 4a), in the parallel direction, real changes are apparent (Fig. 4b). This finding indicates the loss of the long range correlation length as a consequence of the closeness of the blobs. In this context, the long range characteristic length due to the "freezing-in" of the elastic constraints becomes of the same order as the size of the blob, which is produced by the deformation of the random coil surrounding the silica clusters. This is also supported by the loss of fit of the RC model. In fact, at low strain (3\%), for the PDMS10 sample, the three curves of unloaded, loaded in perpendicular, and loaded in parallel direction (not shown) do not present significant variation of the analyzed parameters.

\section{Conclusions}

1. The form factor of these hybrid aerogels is described well by a two-correlation length function based on the Gaussian random coil model. 
2. Clustered elementary particles of inorganic porous silica aerogel of 1-2 nm average size, are surrounded by the organic polymer forming blobs of $6-8 \mathrm{~nm}$ average size, which are mass fractal with dimension of 2.5 .

3. These units are clustered into larger aggregates maintained in equilibrium by the "frozenin" elastic constraints between such units.

4. Butterfly patterns appear when the samples are submitted to uniaxial compression.

5. In the perpendicular direction with respect to the load, no significant structural changes are provoked by the deformation.

6. In the parallel direction with respect to the load, a decrease of the Guinier radius is provoked by the deformation of the random coil surrounding the silica clusters, and the longrange correlation is lost.

\section{Acknowledgments}

This work has been supported in Spain by Project MAT2005-1583 of the CICyT. SANS measurements were made at the HMI, Germany, thanks to the European Commission, under the 6th Framework Programme through the Key Action: Strengthening the European Research Area, Research Infrastructures Contract n : RII3-CT-2003-505925 (NMI3). Authors belong to the Research Group TEP-115 of the Junta de Andalucía. 
Figure 1.

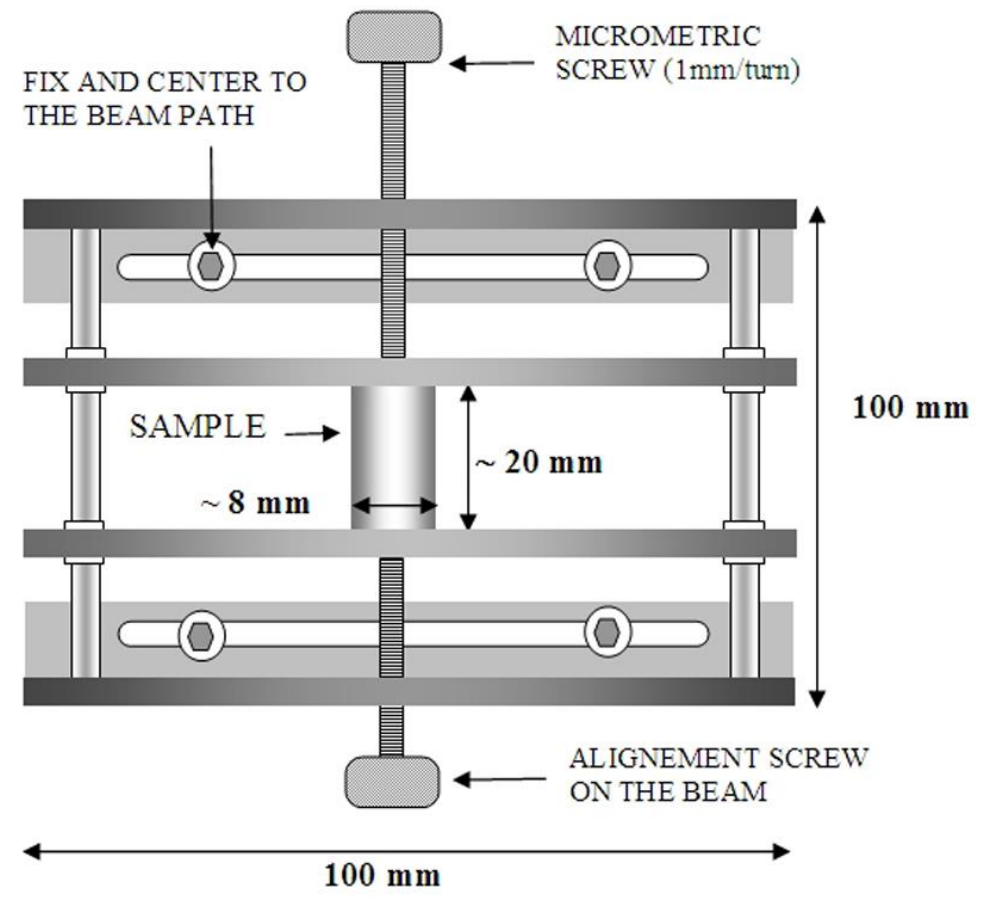


Figure 2.

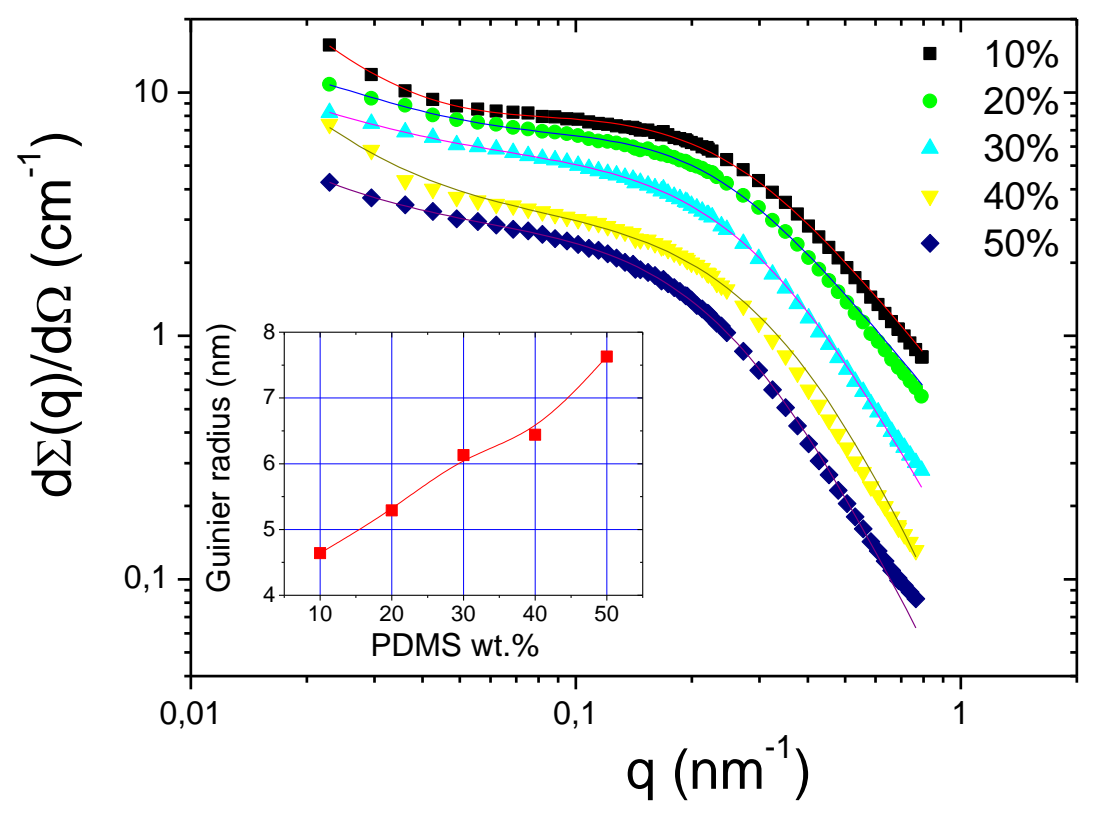


Figure 3.

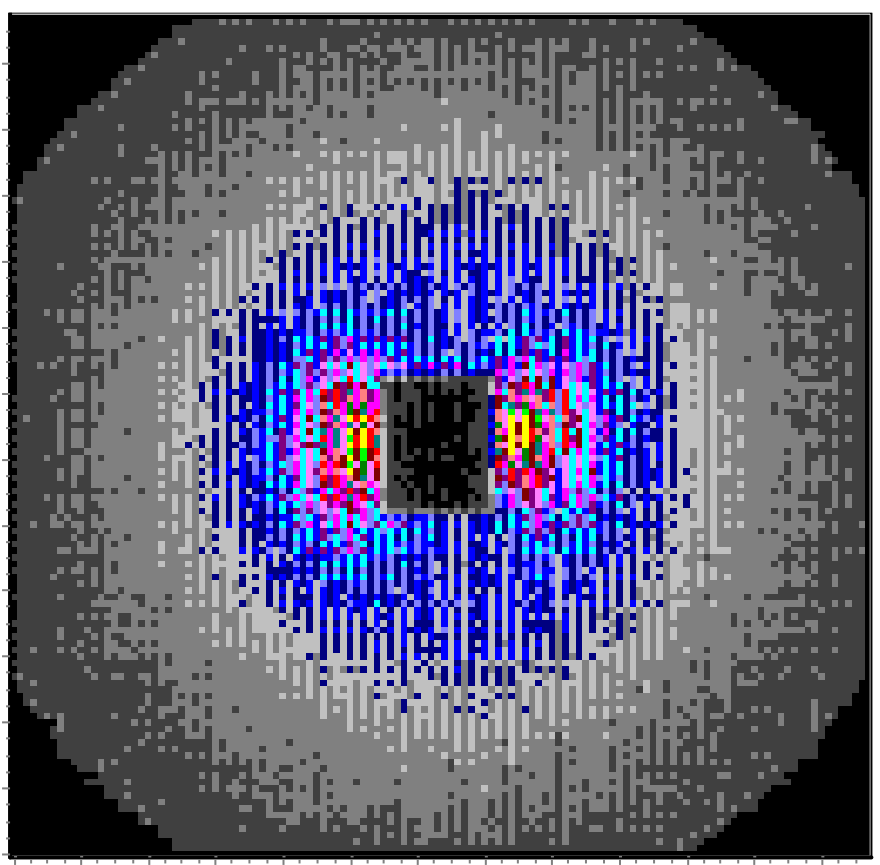


Figure 4.
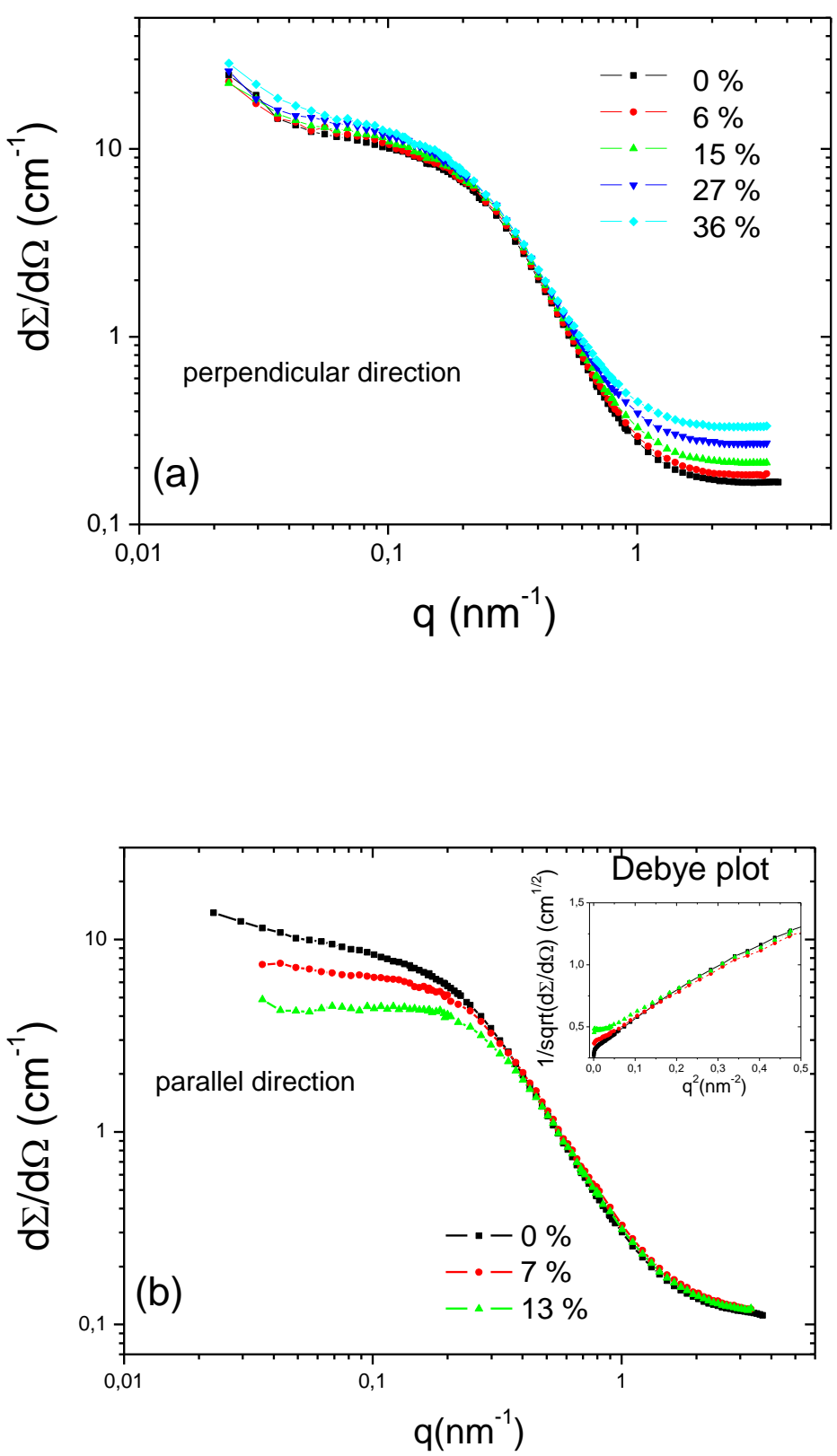


\section{Figure captions:}

Figure 1. Schematic mechanical device for "in situ" measurements of SANS on aerogel samples under compression.

Figure 2. Plot of experimental SANS intensities versus the transfer momentum q, for different PDMS aerogel hybrid samples. Continuous line is the fit to the Gaussian Random Coil model. The corresponding Guinier radius as a function of the polymer content is included in the inset.

Figure 3. Butterfly aspect of the dispersed 2D-intensities in the perpendicular direction with respect to the load during SANS data acquisition.

Figure 4. Plot of SANS intensities versus the transfer momentum q (a) recorded in perpendicular direction with respect to the load, for PDMS40 aerogel under compression at different strains from $0 \%$ to $36 \%$. (b) recorded in parallel direction with respect to the load, for PDMS30 aerogel under compression at different strains from $0 \%$ to $13 \%$. Inset shows its corresponding Debye plot. 
Table 1. Strains of PDMS40 aerogel at different strains and their corresponding Guinier radii values obtained from SANS curves taken in perpendicular $\left(R_{G} \perp\right)$ and parallel $\left(R_{G}||\right)$ directions with respect to the load

\begin{tabular}{c|cc}
\hline PDMS40 & $R_{G} \perp$ & $R_{G} \|$ \\
\hline $0 \%$ & 6.44 & 6.44 \\
$6 \%$ & 6.65 & 5.60 \\
$15 \%$ & 6.70 & 4.12 \\
$27 \%$ & 6.87 & 3.67 \\
$36 \%$ & 7.09 & 3.55 \\
\hline
\end{tabular}




\section{References:}

1 Geissler, E., Horkay, F., Hecht, AM., Rochas, C., Lindner, P., Bourgaux, C. and Couarraze, G., Polymer, 38, (1997), 15-20.

2 Horkay, F., Hecht, AM., Zrinyi, M. and Geissler, E., Polym. Gels and Netw., 4, (1996), 451-465.

3 Schaefer, D.W., Suryawanshi, Ch., Pakdel, P., Ilavsky, J. and Jemian, P.R., Physica A, 314, (2002), 686-695.

4 Schaefer, D.W., Beaucage, G., Loy, D.A., Shea, K.J. and Lin, J.S., Chem. Mater., 16, (2004, 1402-1410.

5 Guo, L., Hyeon-Lee, J. and Beaucage, G., J. Non-Cryst. Solids, 243, (1999), 61-69.

6 Moner-Girona, M., Roig, A., Molins, E., Martínez, E. and Esteve, J., Appl. Phys. Lett., 75, (1999), 653-655.

7 E. Blanco, L. Esquivias, R. Litran, M. Piñero, M. Ramírez-del-Solar and N. de la Rosa-Fox, Appl. Organomet. Chem., 13, (1999), 399-418.

8 Suslick, K.S. and Price, G.J., Annu. Rev. Mater. Sci., 29, (1999), 295-326.

9 McNamara III, W.B., Didenko. Y.T. and Suslick, K., Nature, 401, (1999), 772-775

10 De la Rosa-Fox, N., Esquivias, L., Craievich, A. and Zarzycki, J., J. Non-Cryst. Solids, 121, (1990), 211-215.

11 Mackenzie, J.D., Huang, Q. And Iwamoto, T., J. Sol-Gel Sci. Tech., 7, (1996), 151-161.

12 Piñero, M., Morales-Flórez, V., de la Rosa-Fox, N. and Esquivias, L., Bol. Soc. Esp. Ceram. V., 44(5), (2005), 291-293.

13 Brumberger, H., in “Modern Aspects of Small-Angle Scattering”, NATO ASI series (Vol. 451).Kluwer Academic Publishers, (1995).

14 U. Keiderling, Physica B, 234-236, (1997), 1111.

15 Schaefer, D.W., Brow, R.K., Olivier, B.J. Rieker, T., Beaucage, G., Hrubesh, L. and Lin, J.S., ibid ref. 11, (1995), 299-307.

16 Hoinkis, E., in "Chemistry and Physiscs of Carbon”, Ed. Thrower, P.A., (Vol. 25), Marcel Dekker, Inc. (1997), 72-241.

17 Toledo-Fernández, J. A., Mendoza-Serna, R., Morales-Flórez, V., de la Rosa-Fox, N., Santos, A., Piñero, M., and Esquivias, L., Bol. Soc. Esp. Ceram. V., (2007) (in press).

18 Kirste, R.G. and Oberthur, R.C., in "Small Angle X-ray Scattering”, Ed. Glatter, O. and Kratky, O., Academic Press, (1982), Chap. 12, 387-431.

19 Pedersen, J.S., J. Appl. Cryst., 33, (2000), 637-640.

20 Brinker, C.J. and Scherer, G.W., in "Sol-Gel Science: The Physics and Chemistry of Sol-Gel Processing", Academic Press, Inc., (1990), Chap. 3, 97-228.

21 Debye, P. and Bueche, J., J. Appl. Phys, 20, (1949), 518.

22 Roe, R.-J., in "Methods of X-Ray and Neutron Scattering in Polymer Science“, Oxford University Press, (2000), Chap. 5, 155-208. 